\title{
Movement and fluctuations of the vacuum
}

\author{
Marc-Thierry Jaekel ${ }^{a}$ and Serge Reynaud ${ }^{b}$ \\ (a) Laboratoire de Physique Théorique de l'Ecole Normale Supérieure, \\ Centre National de la Recherche Scientifique, Université Paris-Sud, \\ 24 rue Lhomond, F75231 Paris Cedex 05 France \\ (b) Laboratoire Kastler Brossel, Université Pierre et Marie Curie, \\ Ecole Normale Supérieure, Centre National de la Recherche Scientifique, \\ 4 place Jussieu, Fr5252 Paris Cedex 05 France
}

(LPTENS 97/22)

\begin{abstract}
Quantum fields possess zero-point or vacuum fluctuations which induce mechanical effects, namely generalised Casimir forces, on any scatterer.

Symmetries of vacuum therefore raise fundamental questions when confronted with the principle of relativity of motion in vacuum. The specific case of uniformly accelerated motion is particularly interesting, in connection with the much debated question of the appearance of vacuum in accelerated frames. The choice of Rindler representation, commonly used in General Relativity, transforms vacuum fluctuations into thermal fluctuations, raising difficulties of interpretation. In contrast, the conformal representation of uniformly accelerated frames fits the symmetry properties of field propagation and quantum vacuum and thus leads to extend the principle of relativity of motion to uniform accelerations.

Mirrors moving in vacuum with a non uniform acceleration are known to radiate. The associated radiation reaction force is directly connected to fluctuating forces felt by motionless mirrors through fluctuation-dissipation relations. Scatterers in vacuum undergo a quantum Brownian motion which describes irreducible quantum fluctuations. Vacuum fluctuations impose ultimate limitations on measurements of position in space-time, and thus challenge the very concept of space-time localisation within a quantum framework.

For test masses greater than Planck mass, the ultimate limit in localisation is determined by gravitational vacuum fluctuations. Not only positions in space-time, but also geodesic distances, behave as quantum variables, reflecting the necessary quantum nature of an underlying geometry.
\end{abstract}

\section{INTRODUCTION}

The effects of vacuum fluctuations in microscopic physics have played an essential role in the development of Quantum ElectroDynamics and Quantum Field Theory. Vacuum fluctuations also have an impact on macroscopic physics as they give rise to mechanical forces. Fluctuating forces and motional forces, which generalise the static Casimir forces, have recently been thoroughly examined. It has been shown that vacuum fluctuations affect the notion of movement in vacuum and are directly related to the principle of relativity of motion. They also impose fundamental limitations on localisation and, hence, on the notion of space-time.

The aim of the present paper is to discuss questions connected to these problems. Since a complete and consistent theory of inertial and gravitational phenomena is still lacking, these questions have not yet been satisfactorily answered. We however intend to show that the fluctuations of quantum vacuum have a profound impact on fundamental concepts of relativity and, in particular, that they shed light on the interpretation of space-time localisation in a quantum formalism. We also hope to convince readers that the various physical effects discussed here will have to be considered in any consistent theoretical frame to be developed in the future. In order to put our purpose in perspective, we first briefly recall the historical development of the concept of motion in vacuum.

The notions of void and movement have been associated since the foundation of the atomistic school of philosophy by Leucippus and Democritus. More than two thousand years ago, atoms and void were introduced as complementary and necessary premises to the comprehension of movement. As outlined by Russell [1], this was a logical requirement, more than a physical one, which was intended to answer objections formulated by Zeno against the very possibility of movement. The concept of void played a crucial role since it was allowing motion to take place freely. In this respect, the absence of friction in void was considered from the very beginning as a preliminary requirement for any physics of movement. Although a quite different conception of space, the Aritostelian one, prevailed for a long time, the ideas promoted by the ancient atomistic school were revived by the rise of modern physics.

Galileo introduced the idea that motions with uniform velocity have the same status as rest and that only changes of velocity have to be explained. With this principle of relativity of motion, the absence of resistance to uniform motion was recognized as a condition for the understanding of motion. Shortly after Galileo's time, vacuum became a matter for physical investigation with the experiments of Torricelli, Pascal and von Guericke. Going further along 
the same directions, Newton was able to lay down the fundamental laws of mechanics and gravitation. He emphasized that the observed motions of celestial bodies could only be consistent with negligible friction, so that they must be thought to occur in an empty space. From the exponential decreasing of air density with altitude, Newton estimated that interplanetary space was indeed practically free of matter. Vacuum and the empty space of mechanics were thus identified as the celebrated Newtonian absolute space [2]. From a pragmatic point of view, vacuum could have been qualified at that time as what remains when matter has been removed from a given region in space.

Although the Newtonian conception raised severe difficulties which were immediately noticed, it survived until the advent of Relativity Theory. Meanwhile, empty space was promoted to the status of a universal arena, not only for motion of matter, but also for propagation of fields such as electromagnetic fields. As is well known, the key point of the Einsteinian objection against Newtonian physics is that the properties attributed to empty space by classical mechanics are not compatible with the symmetry properties of Maxwell equations governing electromagnetism [3]. Building a definition of space-time events upon light propagation, Einstein was able to restore the compatibility between mechanics and electromagnetism [4].

The discovery of field fluctuations led to a further enrichment of the notion of vacuum. At any non zero temperature in particular, space is filled with blackbody radiation. It was demonstrated by Einstein that scattering of these thermal field fluctuations damp the motion of any scatterer to rest while giving rise to Brownian fluctuations of position [5]. The close connection between fluctuations and dissipation was thus noticed for the first time. It follows that a more precise definition of vacuum has to be given as what remains when matter and field have been removed from a given region in space.

After having introduced the concept of quanta in physics to build a theory of blackbody radiation [6], Planck modified his law to account for zero-point fluctuations, that is field fluctuations persisting at zero temperature and corresponding to an energy of half a quantum per mode [7]. The status of these fluctuations, quite controversial in a classical formalism [8,9], was more firmly established by the quantum theory of electromagnetic field [10]. Heisenberg inequalities entail a profound modification of the notion of movement since they forbid absolute rest. Position and velocity of a body cannot be simultaneously and precisely fixed and thus possess unsurpassable residual fluctuations. This property subsists in Quantum Field Theory where field modes, each of which is equivalent to an harmonic oscillator, also possess irreducible quantum fluctuations. The fundamental state of the field, defined by the absence of any field excitation, still contains zero-point field fluctuations, also called vacuum fluctuations. The vacuum state may be identified with quantum empty space [11. The principle of relativity of motion in quantum vacuum holds for uniform velocity, as a consequence of Lorentz invariance of vacuum fluctuations [12].

The previous paragraphs sketch a succession of developments which may be compared with the logical line of thought raised by ancient atomism. Movement has to be defined with respect to something. When void is considered as reference for movement, it has thus to be distinguished from nothingness. But if something fills vacuum, it is not clear how it can manage to allow motion without friction. As we have seen, modern physics finally came to a logical answer to this paradox. Vacuum is indeed filled with field fluctuations but these fluctuations do not oppose uniform motion as a consequence of a fundamental symmetry of physics. Lorentz invariance is not only a symmetry of the laws of physics, but also of the vacuum state, that is the quantum version of empty space. The Galilean principle of relativity of motion, that is the preservation of uniform motion in vacuum, has finally been made compatible with a physical description of vacuum. However, inertial and gravitational phenomena still raise essential questions.

The Newtonian laws of inertia deal with accelerated motion and not only with uniform velocity. In order to discuss these laws in the light of the previous arguments, one has to ask two related questions. What is the appearance of vacuum fluctuations for an accelerated body? Does vacuum oppose to accelerated motion? These questions remain nowadays a matter of controversy and will be examined in the present paper. Although there is only one relativistic definition of uniformly accelerated motion, there is a variety of possible representations of uniformly accelerated frames. With the common choice of Rindler representation [13], vacuum is found to be transformed into a thermal bath with a temperature proportional to acceleration and to Planck constant [14 [17]. This correspondance between acceleration and temperature has apparently been easily accepted because of its association with the most spectacular effect predicted by quantum field theory in curved spacetime, namely thermal particle creation due to curvature [18, 19]. It is nevertheless clear that accelerated frames and curved spacetime are completely different physical problems, from the point of view of general relativity.

Decisive progress were brought by explicit computations of scattering of quantum fields by perfect mirrors moving in vacuum 20,21]. Mirrors moving in vacuum with a non uniform acceleration were predicted to radiate [22]. In the historical context recalled previously, this means that motion in quantum vacuum gives rise to a friction force. It has however to be emphasized that this force vanishes in the specific cases of uniform velocity or uniform acceleration. This result was understood as challenging the alleged equivalence between accelerated vacuum and thermal bath. Although it remains logically compatible with it, it points to the difficulty of finding an uncontested physical significance to this equivalence. This is not so surprising if one realises that Rindler transformations do not preserve Maxwell laws. After such a transformation, light rays appear to be curved while frequencies undergo a redshift during propagation 
[23]. Moreover, uniform velocity and rest do not play the same role after these transformations. In other words, the Rindler representation of accelerated frames significantly departs from the point of view of invariance and symmetry properties which made the success of Lorentz transformations [4]. This point of view may however be applied also for uniformly accelerated frames provided the latter are represented through conformal coordinate tranformations [24, 25] which fit the symmetry properties of electromagnetic theory and of quantum vacuum.

The existence of vacuum field fluctuations has been recognised for a long time and their effects thoroughly studied [26]. The possibility of manipulating vacuum fluctuations and, even, to squeeze their effect on quantum noise in optical measurements has been demonstrated 27 30]. As far as macroscopic effects of vacuum fluctuations are concerned, the investigation of static Casimir forces [31]33] has led to an experimental demonstration of vacuum radiation pressure [34 38]. The closely related effect of attraction of an atom to a conducting plate has also been demonstrated [39]. However, vacuum fluctuations remain a matter of debate mainly because their energy is infinite. More strikingly, their energy per unit volume is infinite. This problem may be considered as a particular occurence of well known formal difficulties of Quantum Field Theory. It raises a specific question concerning gravitational theory since energy is the source of gravity in General Relativity [40]. As most situations within Quantum Field Theory only involve differences of energy, vacuum energy may be considered to be zero by definition. The energy of field states is then determined relatively to vacuum. In fact, this procedure does not answer the question. The Casimir energy, which is a difference between energies of two vacuum configurations, has to contribute to gravity. It is also known that the definition of vacuum depends on the choice of coordinates and does not meet the covariance requirement of General Relativity [16].

It has sometimes been argued that these problems can be solved, at least from a pragmatical point of view, if the microscopic and macroscopic domains are clearly separated [41]. Taken seriously, this argument means that fundamental laws of physics hold at the elementary level of microscopic physics where we have to renounce to general principles of mechanics, while the mechanical description of nature has a validity restricted to the macroscopic domain. This argument is known to be conceptually inconsistent and to endanger the consistency of quantum formalism because it mixes too crudely quantum and classical descriptions [12]. It has now lost its pragmatical pertinence, due to the progress towards highly sensitive measurements of macroscopic objects 443, 44. Since the quantum level of sensitivity is approached for such measurements, it appears more and more uneasy to delineate any a priori frontier between microscopic and macroscopic domains. Moreover, the fluctuations of the stress tensor corresponding to quantum matter and quantum field present in space-time must induce fluctuations of the space-time metric itself [45]. These arguments contribute to render a classical conception of space-time untenable in the presence of quantum fields and plead for a definition of localisation accounting for quantum fluctuations.

The standard treatment of Quantum Field Theory can also be applied to gravitational fields [46 50]. It has however been early recognised that the infinities generated by radiative processes due to gravitational fields cannot be dealt with usual renormalisation procedures [51]. A new theory of Quantum Gravity is needed in order to describe all interactions including gravitation. Since such theory is not yet available, discussions of the influence of quantum fluctuations on the general principles associated with relativity have remained preliminary and limited by approximations which remain uncontrolled by necessity. In most approaches, Einstein's General Relativity is considered as a starting basis for a theory of gravitation which must remain valid at low energies. Due to quantum fluctuations of gravitation, profound modifications are expected at energies comparable to Planck energy, which should affect the ultimate structure of space-time at very short distances [52]. The interpretation of gravitation as geometric property of space-time, which is inherited from the classical theory, has to find a new formulation in quantum theory [53,54]. The question of the nature of a quantum vacuum including gravitation and, hence, of a space-time being an arena for all interactions still remains an open problem. In the present paper, we shall try to provide hints for the construction of such a quantum framework, by emphasizing the necessity of a consistency between vacuum field fluctuations and the property of relativity of motion.

\section{RELATIVITY OF MOTION IN VACUUM}

Modern mechanics is founded on the principle of inertia, that is the conservation of uniform motion in the absence of external forces. This property may equivalently be stated as relativity of uniform motion in empty space. In particular rest is not a privileged state of motion. With the advent of quantum theory, empty space is the place of vacuum fluctuations and the symmetry of vacuum determines whether or not the principle of relativity of motion remains valid for motion in quantum empty space.

In order to study the effect of motion on vacuum fields, one has first to represent motion. Within the formalism of Quantum Field Theory, motion is usually reduced to a mapping of space-time coordinates fitting a classical trajectory in space-time. Arbitrary motion is represented by this technique, which however relies on the same classical approxi- 
mation used when curved space is represented by a classical background metric [16, 17]. This approach perfectly fits the covariance techniques of General Relativity. In the quantum regime however, it is known to generate paradoxes due to a too crude mixing between quantum effects and classical descriptions of motion 42].

A second approach puts emphasis on invariance properties in the spirit of Special Theory of Relativity [3]. Since it is based upon symmetry properties associated with space-time transformations, this approach is limited to particular motions. As an emblematic example of this approach, the principle of relativity of uniform motion is identified with the Lorentz invariance of Maxwell equations [4]. In Quantum Field Theory, the connection between vacuum fluctuations and field propagation ensures that the electromagnetic vacuum also satisfies invariance under Lorentz transformations. In the present section, we shall see that the conformal invariance of electromagnetism allows to deal with uniformly accelerated motion in the same manner as Lorentz invariance for uniform motion [55,56].

As a first step,vacuum, which is the ground state, is characterised as the particular case of thermal equilibrium state corresponding to a zero temperature. For such a stationary state, different correlation functions can be defined for any two fields $A$ and $B$

$$
\begin{aligned}
C_{A B}(x) & =<A(x) B(0)>-<A(x)><B(0)>=\hbar \sigma_{A B}(x)+\hbar \xi_{A B}(x) \\
2 \hbar \sigma_{A B}(x) & =C_{A B}(x)+C_{B A}(-x) \quad 2 \hbar \xi_{A B}(x)=C_{A B}(x)-C_{B A}(-x)
\end{aligned}
$$

The commutator $\xi_{A B}$ exhibits the quantum character of fields $A$ and $B$, while the anticommutator $\sigma_{A B}$ describes their fluctuations. These functions are more easily manipulated in the Fourier domain. When using the generic definition

$$
f(x)=\int \frac{d^{4} k}{(2 \pi)^{4}} e^{-i k \cdot x} f[k]
$$

$\xi_{A B}[k]$ is identified with a field propagator and $\sigma_{A B}[k]$ with a field noise spectrum. At thermal equilibrium, correlation functions satisfy fluctuation-dissipation relations [57,58] which are just a general expression of the Planck law [7]

$$
C_{A B}[k]=\frac{2 \hbar \xi_{A B}[k]}{1-e^{-\beta \omega}} \quad \sigma_{A B}[k]=\operatorname{coth}\left(\frac{\beta \omega}{2}\right) \xi_{A B}[k]
$$

where $\omega$ is the field frequency $k_{0}, \beta=\frac{\hbar}{k_{B} T}$ characterises temperature $T$ while $\hbar$ and $k_{B}$ represent respectively Planck and Boltzmann constants. For the sake of simplicity, the velocity of light $c$ is taken as unit velocity. However, we have chosen to write it explicitly in a few equations which may be used to evaluate orders of magnitude. The zero temperature limit of equations (3) gives relations characteristic of vacuum ( $\theta$ is Heaviside's step function and $\epsilon$ is the sign function)

$$
C_{A B}[k]=2 \hbar \theta(\omega) \xi_{A B}[k] \quad \sigma_{A B}[k]=\epsilon(\omega) \xi_{A B}[k]
$$

For instance, vacuum fluctuations of the electromagnetic potential $A_{\mu}$ may be described by any one of the equivalent expressions

$$
\begin{array}{rlrl}
C_{A_{\mu} A_{\nu}}[k] & =2 \hbar \theta(\omega) \xi_{A_{\mu} A_{\nu}}[k] & \xi_{A_{\mu} A_{\nu}}[k]=\pi \delta\left(k^{2}\right) \eta_{\mu \nu} \\
C_{A_{\mu} A_{\nu}}(x) & =\frac{\hbar}{\pi} \eta_{\mu \nu} \frac{1}{\left(x_{0}-i \varepsilon\right)^{2}-\mathbf{x}^{2}}
\end{array}
$$

where $\eta_{\mu \nu}$ is Minkowski metric, i.e. a diagonal matrix with elements $(1,-1,-1,-1)$ on the diagonal, and $\delta$ is Dirac's measure; $x_{0}$ is the time delay and $\mathbf{x}^{2}$ the squared spatial distance between the two points where fields are evaluated $\left(\varepsilon \rightarrow 0^{+}\right.$, and Feynman gauge has been used). Vacuum correlations of electromagnetic fields are thus characterised by a spectrum limited to the light cone and to positive frequencies. The spectrum is furthermore invariant under Lorentz transformations. Conversely, the spectrum of vacuum field fluctuations is completely determined by these properties, up to a constant factor proportional to $\hbar$. Planck constant can be considered as fixing the scale of vacuum or zero-point fluctuations [7,12] which correspond to an energy of $\frac{1}{2} \hbar \omega$ per mode of frequency $\omega$.

Invariance of vacuum under Lorentz transformations ensures that it has the same appearance for all uniformly moving observers. The extension to uniformly accelerated observers is confronted to the problem of finding a representation for uniformly accelerated frames. As already noticed, there exists a variety of possible representations which are all in agreement with the same definition of uniformly accelerated motion [59]. As a matter of fact, vacuum fluctuations depend on the global definition of frame transformations and not only on the definition of a single classical trajectory. Infinitely many different changes of frame can be used to transform a given accelerated trajectory to rest. A common choice within the framework of General Relativity is to use Rindler coordinates [13 which are designed such that they transform rest into uniformly accelerated motion while preserving the rigidity of bodies. Paradoxical 
properties arise when discussing the transformation of quantum fields, since the sign of frequencies is not preserved. This means that the number of particles and even the distinction between particle and antiparticle is not the same for different uniformly accelerated observers within this formalism. In particular, vacuum is not invariant, but is transformed into a thermal bath with a temperature proportional to the acceleration $a$ [15]

$$
\frac{\beta a}{c}=\frac{\hbar a}{k_{B} T c}=2 \pi
$$

Paradoxes and conflicting views are raised by this result. On one hand, the detection of thermal quanta by a detector moving in vacuum with uniform acceleration has been predicted, using a simplified model of detector. However, the detection process in the moving frame has to be interpreted differently by an observer at rest, for whom the excitation of the detector is accompanied by the emission of a quantum. This leads to conflicts with the principle of energy conservation, the solution of which require subtle reasoning [60,61]. A few suggestions have been made for the observation of the modification of vacuum by acceleration 62 64. On the other hand, it has also been argued that an oscillator moving with uniform acceleration in vacuum does not radiate 65] neither detects thermal quanta 666. The paradoxes raised by detection have also been discussed by modelling the detector as a two level atom [67. The consequences for the equivalence principle of the transformation of vacuum into a thermal bath have been discussed, leading to the introduction of different vacua depending on the physical situation and the observer [68]. In view of these difficulties, two simple points may be recalled here. First, Rindler transformations do not preserve Maxwell equations. In other words, the field propagator is changed under such a transformation. In these conditions, it is not so surprising to obtain a change of the vacuum spectrum or of the relation between the vacuum spectrum and the propagator. Then, a thermal bath has quite different physical properties than vacuum. It leads to a friction force for uniform motion so that uniform velocity is no longer equivalent to rest in a Rindler frame. But there is no simple composition rule for different Rindler transformations or even for Rindler transformations and Lorentz transformations. Rindler transformations do not form a group.

Another representation of uniformly accelerated frames can be used which is more adapted to the point of view of symmetry properties. Shortly after Einstein built up the Special Theory of Relativity, Bateman and Cunningham 24.25] established that Maxwell equations possess a symmetry group larger than the Poincaré group of translations and Lorentz transformations. This group contains conformal coordinate transformations defined as those transformations for which the modified metric keeps the form of Minkowski metric, up to a conformal factor. The group may be generated by Poincaré transformations and the transformations of space-time coordinates $x^{\mu}$ which can be built with translations and four-dimensional inversion and which are defined by

$$
x^{\mu} \rightarrow \bar{x}^{\mu}, \quad \frac{\bar{x}^{\mu}}{\bar{x}^{2}}=\frac{x^{\mu}}{x^{2}}-a^{\mu}
$$

where $a^{\mu}$ plays the role of an acceleration four-vector. These transformations may be identified as transformations to accelerated frames. To be more precise, uniformly accelerated motion may be defined in Relativity Theory by the condition that the Abraham vector vanishes [59]. This vector $\Gamma_{\mu}$ is defined, for any trajectory $x_{\mu}(\tau)$, as $(\tau$ is a proper time defined along the trajectory)

$$
\Gamma^{\mu}=\frac{d^{3} x^{\mu}}{d \tau^{3}}+\left(\frac{d^{2} x}{d \tau^{2}}\right)^{2} \frac{d x^{\mu}}{d \tau} \quad d \tau=\sqrt{\eta_{\mu \nu} d x^{\mu} d x^{\nu}}
$$

Conformal transformations preserve the Abraham vector (8) up to a factor [69]. Hence, they also preserve the definition of uniformly accelerated motion. This means that rest is transformed into uniformly accelerated motion under a conformal transformation and, conversely, that a conformal transformation can be found which transforms a given uniformly accelerated motion into rest. For that reason, it has been possible to use conformal transformations 70 72] to discuss the relativistic effects of uniform acceleration [23].

Since Maxwell equations are invariant under conformal transformations, the propagator of electromagnetic fields is also preserved and it is worth studying the transformation of vacuum correlation functions. It has in fact been demonstrated that these functions are also invariant [55. The noise spectrum $\sigma_{F_{\mu \nu} F_{\rho \sigma}}$ which characterises the fluctuations of the electromagnetic field

$$
F_{\mu \nu}=\partial_{\mu} A_{\nu}-\partial_{\nu} A_{\mu}
$$

has exactly the same form in the accelerated frame when written in terms of the transformed coordinates as in the inertial frame when written in terms of the original coordinates. The noise spectrum $\sigma_{A_{\mu} A_{\nu}}$ which characterises the fluctuations of the electromagnetic potential has its form changed but this change may be eliminated by gauge 
transformation. Similarly, Maxwell equations written in terms of the electromagnetic potential are invariant up to a gauge transformation $[73,74]$.

We may emphasize that correlation functions are written in the conformal accelerated frame as well as in the inertial frame in terms of a Minkowski metric. Hence, the preservation of vacuum fluctuations is not a covariance statement which would merely mean that the change of form due to a coordinate transformation is compensated by the corresponding change of the metric. This preservation should rather be interpreted as an invariance property under conformal transformations, which generalises the known invariance property under Poincaré transformations.

In order to emphasize the symmetry properties associated with conformal invariance, it is worth introducing the conformal algebra [75] associated with the group formed by conformal coordinate transformations. The generators of this algebra include Lorentz generators $J_{\mu \nu}$ and translations $\left(P_{\mu}\right)$ which form the Poincaré algebra

$$
\begin{array}{lr}
\left(P_{\mu}, P_{\nu}\right)=0 \quad\left(J_{\mu \nu}, P_{\rho}\right)=\eta_{\nu \rho} P_{\mu}-\eta_{\mu \rho} P_{\nu} \\
\left(J_{\mu \nu}, J_{\rho \sigma}\right)=\eta_{\nu \rho} J_{\mu \sigma}+\eta_{\mu \sigma} J_{\nu \rho}-\eta_{\mu \rho} J_{\nu \sigma}-\eta_{\nu \sigma} J_{\mu \rho}
\end{array}
$$

as well as a dilatation generator $(D)$ and four generators $\left(C_{\mu}\right)$ for transformations to accelerated frames (7) satisfying the following commutation rules

$$
\begin{aligned}
& \left(D, P_{\mu}\right)=P_{\mu} \quad\left(D, J_{\mu \nu}\right)=0 \\
& \left(P_{\mu}, C_{\nu}\right)=-2 \eta_{\mu \nu} D-2 J_{\mu \nu} \\
& \left(J_{\mu \nu}, C_{\rho}\right)=\eta_{\nu \rho} C_{\mu}-\eta_{\mu \rho} C_{\nu} \\
& \left(D, C_{\mu}\right)=-C_{\mu} \quad\left(C_{\mu}, C_{\nu}\right)=0
\end{aligned}
$$

The total number $N$ of photons is invariant under all these conformal transformations

$$
\left(P_{\mu}, N\right)=\left(J_{\mu \nu}, N\right)=(D, N)=\left(C_{\mu}, N\right)=0
$$

As a particular case, the vacuum state defined by $N=0$ is invariant under conformal transformations. More generally, the notion of $N$ photon states is the same for inertial or uniformly accelerated observers. This allows to discuss how the frequency of a photon is changed in a transformation to accelerated frames and, hence, to establish a connection between Einstein equivalence principle and the domain of quantum fluctuations [56].

Conformal invariance allows a simple discussion of scattering of vacuum fields by uniformly accelerated mirrors. A scatterer uniformly accelerated in vacuum may indeed be transformed into a scatterer at rest through a conformal transformation which leaves vacuum invariant. In other words, vacuum as defined for an inertial observer remains vacuum for a uniformly accelerated observer. As an immediate consequence, a mirror moving with uniform acceleration in vacuum behaves like a mirror at rest and does not radiate. The absence of radiation may be stated as the preservation of relativity of motion for uniformly accelerated motion in vacuum [55].

This result has in fact been obtained in a number of specific cases by explicit computation. Fulling and Davies were the first ones to establish that the force experienced by a perfect mirror moving in a two-dimensional scalar vacuum exactly vanishes for uniformly accelerated motion [22]. This result was extended to the case of plane mirrors moving in four-dimensional space-time, in conformally invariant scalar fields [76] or electromagnetic fields [77. For all these calculations, the free quantum field theory obeys conformal invariance and the scattering upon the perfect mirror also obeys conformal invariance. The general result reported in the present section has a larger domain of validity since the second condition does not appear to be necessary. Actually, the absence of radiation for uniformly accelerated motion has also been established for scatterers which do not obey conformal invariance like partly transmitting mirrors where a frequency scale is given by the reflection cutoff [78] and Fabry-Perot cavities where a length scale is given by the distance of the two mirrors [79].

\section{DISSIPATIVE MOTION IN VACUUM}

The presence of quantum field fluctuations implies that scatterers are submitted to a fluctuating radiation pressure, even when they are at rest in vacuum [80]. Preservation of inertial motion in vacuum corresponds to the conservation of the mean values of energy and momentum but it is also a consequence of conservation laws that a scatterer at rest in vacuum feels a fluctuating force [78]. These force fluctuations and the associated dissipation are analysed in detail in this section, which begins by discussing the fluctuations of the vacuum stress tensor which determine the radiation pressure exerted by electromagnetic fields upon scatterers.

Maxwell stress tensor $T_{\mu \nu}$ has vacuum correlations which may be determined from those of the electromagnetic field (5) 


$$
\begin{gathered}
C_{T_{\mu \nu} T_{\rho \sigma}}[k]=\frac{\hbar^{2}}{40 \pi} \theta(\omega) \theta\left(k^{2}\right)\left(k^{2}\right)^{2} \pi_{\mu \nu \rho \sigma} \\
\pi_{\mu \nu \rho \sigma}=\frac{1}{2}\left(\pi_{\mu \rho} \pi_{\nu \sigma}+\pi_{\mu \sigma} \pi_{\nu \rho}\right)-\frac{1}{3} \pi_{\mu \nu} \pi_{\rho \sigma} \quad \pi_{\mu \nu}=\eta_{\mu \nu}-\frac{k_{\mu} k_{\nu}}{k^{2}}
\end{gathered}
$$

Like for vacuum correlations of fields, the spectra are completely determined in vacuum by their symmetry properties, up to a numerical factor. Indeed, stress tensors are symmetric in their two indices, while conformal symmetry results in a vanishing trace. Energy-momentum conservation implies that divergence of the stress tensor vanishes, so that its tensorial form is completely determined. Lorentz invariance limits energy-momentum dependence to Lorentz scalars, and to the interior of the light cone and positive frequencies. Finally, fluctuations of quadratic forms in the fields must scale like $\hbar^{2}$. Radiation pressure fluctuations thus take a form which is determined by the symmetries due to the conservation laws and the constraints associated with vacuum 80,81.

The fact that a scatterer is submitted to a fluctuating radiation pressure in vacuum, even when it stays at rest, signals the existence of forces opposing to motion of the scatterer. As emphasized in the Introduction, the existence of vacuum fluctuations drastically affects the problem of motion in empty space. When a scatterer is set into motion, the quantum fields which are scattered are perturbed and this perturbation modifies energy-momentum balance. Energy-momentum is exchanged between field and scatterer, leading to a reaction force exerted by vacuum fields on the mirror. The radiation reaction force has first been studied within the formalism of Quantum Field Theory by considering mirrors as perfectly reflecting boundaries for the quantum field [21]. In the case of a scalar field scattered by a perfect mirror in a two-dimensional space-time, the radiation reaction force has been shown to be proportional to the Abraham vector (8) for any mirror's trajectory [22]

$$
F^{\mu}=\frac{\hbar}{6 \pi c^{2}} \Gamma^{\mu}
$$

Radiation reaction vanishes for uniform acceleration, due to conformal symmetry of vacuum. This expression also shows that non uniform acceleration is accompanied by radiation. This approach may be applied to arbitrary motions, but it is limited to perfect mirrors and is plagued with infiniteness problems well-known from the study of Casimir energy.

In early derivations [31], Casimir energy was interpreted as a modification of field eigenfrequencies, and hence of the zero-point energy, due to the presence of boundaries. Once two reflectors are present, vacuum energy depends on their distance, giving rise to mean Casimir forces. This approach had to face the infiniteness of vacuum energy and formal prescriptions were introduced to obtain finite results [16]. It was however suggested that a more satisfactory solution would be to suppose mirrors to be transparent at the limit of high frequencies. This is certainly a reasonable assumption for any realistic mirror which, furthermore, eliminates the infiniteness problem. After Casimir forces were given a local interpretation as due to the radiation pressure of vacuum fields [82], a scattering matrix approach was developed to deal with frequency-dependent reflectivities. This approach allowed to obtain finite forces without encountering any infinite quantity [83]. It has also been used to study the case of narrow-band dielectric mirrors and to compute Casimir forces limited by the mirrors' scattering bandwidths 84,85. This approach was then extended to the case of moving mirrors by making use of the linear response formalism [78]. It is thus restricted to motions of small amplitude, but it allows to show that the force induced in reaction to motion is related to the force fluctuations felt by mirrors at rest through general fluctuation-dissipation relations.

Relations between fluctuations and dissipation were first established by Einstein, in the case of Brownian motion in a thermal bath [5]. From energy-momentum conservation and thermal equilibrium, Einstein showed that the Brownian diffusion of momentum is accompanied by a dissipative force opposed to the scatterer's motion and characterised by a friction coefficient depending on the diffusion coefficient and the bath temperature. Fluctuation-dissipation relations were first given their full quantum form by Callen and Welton [57] and later generalised to the linear response formalism by Kubo [58]. The radiation reaction force can be seen to emerge quite generally as a particular kind of causal response function. For motions of small amplitude, the linear response formalism can be applied and the derivation of the reaction force can be briefly sketched as follows. Whatever the Hamiltonian of the total system is, it can be decomposed into free and interacting parts

$$
H=H_{0}+H_{I} \quad \delta H_{I}=-\delta q(t) F(t)
$$

The latter part describes the interaction between the field and the sources on the mirror, which is perturbed by the motion of the scatterer. At first order, this perturbation is proportional to the scatterer's displacement $\delta q$ and its generator is identified as the force $F$ felt by the scatterer [78]. This relation can also be seen as the result of transformations under displacements generated by the field momentum, provided the interaction is translation invariant, i.e. the total energy and momentum are conserved. 
The linear response of any function $A$ of the interacting fields to the perturbation is then described by a linear susceptibility which is directly related to the commutator of this function with the generator

$$
\begin{gathered}
<\delta A(t)>=\int_{-\infty}^{\infty} d t^{\prime} \chi_{A F}\left(t-t^{\prime}\right) \delta q\left(t^{\prime}\right) \quad<\delta A[\omega]>=\chi_{A F}[\omega] \delta q[\omega] \\
\operatorname{Im}\left(\chi_{A F}\right)[\omega]=\xi_{A F}[\omega]
\end{gathered}
$$

In other words, the dissipative part of the susceptibility coincides with the commutator spectrum. The reactive part of the susceptibility may be reconstructed from the dissipative part through dispersion relations, but short time singularities might require that subtractions are accounted for in this reconstruction 86. Fluctuation-dissipation relations (16) characterise the linear response of a quantum system to a perturbation 58]. At thermal equilibrium, the commutator spectrum $\xi_{A B}$ is directly related to correlation functions [57]. At zero temperature in particular, the relations (4) characteristic of vacuum fluctuations are obtained.

Clearly, the motional susceptibility of the force itself is related to the fluctuations of the force exerted on the scatterer at rest, according to (16)

$$
\begin{aligned}
& <\delta F[\omega]>=\chi_{F F}[\omega] \delta q[\omega] \\
& C_{F F}[\omega]=2 \hbar \theta(\omega) \xi_{F F}[\omega]=2 \hbar \theta(\omega) \operatorname{Im}\left(\chi_{F F}[\omega]\right)
\end{aligned}
$$

To illustrate these properties, let us consider the simple case of a perfect mirror scattering a scalar field in a two-dimensional space-time. In that case, stress tensor fluctuations (13) lead to the following fluctuations for the momentum density $p$ of incoming fields at an arbitrary point

$$
C_{p p}[\omega]=\frac{\hbar^{2}}{12 \pi} \theta(\omega) \omega^{3}
$$

For a perfect mirror, all incoming momentum is reflected back and, hence, the radiation pressure is twice the incoming momentum density

$$
C_{F F}[\omega]=\frac{\hbar^{2}}{3 \pi c^{2}} \theta(\omega) \omega^{3}
$$

Hence, a force proportional to the third time derivative of the mirror's position is obtained [78]

$$
\xi_{F F}[\omega]=\frac{\hbar}{6 \pi c^{2}} \omega^{3} \quad<\delta F(t)>=\frac{\hbar}{6 \pi c^{2}} \delta q^{\prime \prime \prime}(t)
$$

This is the dissipative force (14) for a perfect mirror moving in vacuum 22 at the limit where the velocity remains much smaller than the velocity of light.

The dissipative force felt by perfect mirrors moving in four-dimensional space-time and in vacuum of different quantum fields have similarly been computed and shown to involve higher derivatives of the mirror's position $76,87,87$. As in the two-dimensional case, they are connected through fluctuation-dissipation relations with force fluctuations evaluated for three-dimensional mirrors which could have different shapes 80, 88, 89. This connection has also been established for mirrors described by frequency-dependent scattering matrices [78]. At this point, it is worth discussing the analogy with the problem of radiation reaction in ElectroDynamics. It has been known for long [90] that a charge moving in vacuum with non uniform acceleration radiates and that the loss of energy-momentum gives rise to the Abraham-Lorentz radiation reaction force which is proportional to Abraham vector (8). The equation of motion for a charge in vacuum has also been written as a Quantum Langevin equation, with the radiation reaction force and the fluctuating Langevin force satisfying fluctuation-dissipation relations [91,92, in close analogy with relations (20). The fact that electric charges and dipoles feel a fluctuating vacuum field, the properties of which are characterized by a noise spectrum proportional to $\omega^{3}$, has been thoroughly demonstrated by studies of spontaneous emission processes 93, 94. It is also known from discussions of classical electron theory, that an equation of motion involving third derivatives leads to instabilities or violations of causality [95. In the case of mirrors, this difficulty points to inconsistencies of the model of perfect mirror which can be circumvented by considering mirrors transparent at field frequencies higher than a cut-off frequency. Under the condition that the energy corresponding to the cutoff is smaller than the mass of the mirror, the causal scattering of the field and the characteristic properties of vacuum spectra lead to a mirror's susceptibility which is itself a passive, and hence causal, function 96]. This condition for causal motions is violated by perfect mirrors. In fact, the renormalisation procedure used for perfect mirrors, as well as for electrons, to render finite an infinite mass, is incompatible with a causal mechanical description [91]. 
The modelisation of mirrors as point-like structures does not give account of important physical consequences of spatial extension. Cavities built with two mirrors appear as the simplest systems to discuss such consequences. A lot of discussions has been devoted to the production of radiation inside a cavity built with two perfectly reflecting mirrors [97.98]. However, these calculations do not consider the photons radiated by the cavity which is treated like a closed system. Even for the photons produced inside the cavity, they disregard the important problem of finite lifetime of photons inside the cavity. When built with partly transmitting mirrors in contrast, the cavity appears as an open system able to radiate into the free field vacuum. Moreover, exploiting the finesse of the cavity to amplify the emitted radiation may bring dissipation of motion in vacuum within the realm of experimental observation [99, 100]. Two mirrors in vacuum not only feel the static Casimir force, but also fluctuating forces. They also undergo dissipative forces when they are set into motion, since the fields scattered by the two mirrors are affected by their motion. As in the case of a single mirror, the motional Casimir forces $F_{i}$ can be obtained at first order in the mirrors' displacements $\delta q_{j}$ in terms of linear susceptibilities [101]

$$
<\delta F_{i}[\omega]>=\sum_{j} \chi_{F_{i} F_{j}}[\omega] \delta q_{j}[\omega]
$$

The motional susceptibilities are related through fluctuation-dissipation relations to the fluctuations of Casimir forces felt by the mirrors at rest.

The mechanical susceptibilities can be first studied for very slow motions, i.e. in the quasistatic limit where the motional susceptibilitites are dominated by reactive contributions. At zero frequency, these contributions include the variation of the Casimir force with the mirror's distance. For low frequencies, they also contain terms which correspond to modifications of the inertial responses of the mirrors. These inertial forces show remarkable relativistic properties. They include not only mass corrections for each mirror, depending on the mirrors' distance, but also a force acting on a mirror and depending on the other's acceleration. When taken altogether and for a global motion of the cavity, the resulting inertial correction corresponds to the inertia of the Casimir energy of the cavity. Hence, inertial forces emerge for a cavity with finite spatial extension, although no radiation is predicted, in consistency with the conformal invariance of vacuum. This also shows that the law of inertia of energy still holds for energies due to vacuum fluctuations [79]. The energy due to the Casimir forces can furthermore be seen as an energy stored inside the cavity, as a result of the scattering time delays undergone by field fluctuations. Like the radiation pressure, the field energy inside the cavity fluctuates. As a consequence, the inertial mass of the cavity in vacuum has its proper quantum fluctuations 102.

Quite generally, the susceptibilities (21) involve the same Airy function which determines the energy spectral density inside the cavity [103]. As a result, motional Casimir forces are resonantly enhanced when the mechanical frequency $\omega$ associated with motion is an integer multiple of optical resonance frequencies of the cavity [100]

$$
\omega=n \frac{\pi}{\tau} \quad n \geq 2
$$

$\tau$ is the field propagation time from one mirror to the other. At resonance, the displacements of the mirrors also induce an important redistribution of the energy density inside the cavity, leading to the formation of energy peaks [104,105. As for a single mirror, the motions of the mirrors of the cavity induce a radiation, but the latter is now enhanced for resonant motions. Let us consider the case of two mirrors following harmonic motions at a frequency $\omega$ and with an amplitude $a$. For a long oscillation time $T$ and a cavity with a high finesse $\mathcal{F}$, the number $N$ of radiated photons is found to be proportional to the product of three dimensionless numbers

$$
N \simeq \frac{\omega T}{2 \pi} \frac{v^{2}}{c^{2}} \mathcal{F} \quad v \simeq \omega a
$$

The first factor is the number of mechanical oscillation periods during the time $T$ and the second one is the square of the peak velocity $v / c$ of the vibrating mirrors. These two first factors represent the result expected for a single vibrating mirror, and the corresponding order of magnitude is vanishingly small for any reasonable material velocity. The third factor represents a resonance enhancement proportional to the finesse and allows to hope that dissipation of motion in vacuum may be brought within the realm of experimental observation, provided very high finesses are used 100. In equation (23), the mechanical oscillation frequency has been supposed to be equal to one of the resonance frequencies (22). Precisely, even modes $\omega=\frac{2 \pi}{\tau}, \frac{4 \pi}{\tau} \ldots$ correspond to elongation modes with a periodic modulation of the mechanical cavity length while odd modes $\omega=\frac{3 \pi}{\tau}, \frac{5 \pi}{\tau} \ldots$ are excited by a global translation of the cavity with its length kept constant. For the latter effect as well as in the case of a single oscillating mirror, the cavity moves in vacuum without any further reference than vacuum itself. For the cavity however, radiation is now enhanced by the cavity finesse. We may emphasize that the number of radiated photons diverges at the limit of perfectly reflecting mirrors where the finesse goes to infinity. This shows once more that the simple model which represents mirrors as perfect reflectors and, hence, the cavity as a closed system misses important physical phenomena. 


\section{VACUUM FLUCTUATIONS AND LOCALISATION}

The existence of quantum fluctuations leads to reconsider the notion of position and, hence, of motion which can be considered as a set of different positions in time. Quantum Mechanics has early been known to modify the concept of position to account for the incompatibility between a definite position and a definite velocity. Heisenberg inequalities constrain position and velocity uncertainties in a given quantum state and connect them to the commutator between position and momentum. These inequalities also provide a standard quantum limit for measurements of position 106108 .

The sensitivity limits are more precisely analysed in terms of noise spectra, which describe the quantum fluctuations associated with non commuting variables [109]. This technique has been thoroughly used in Quantum Optics [30]. It also allows to discuss quantum fluctuations of position within the framework of Quantum Field Theory. As a consequence of quantum force fluctuations, scatterers in vacuum undergo a quantum Brownian motion 110. Positions have fluctuations related to force fluctuations and, in particular, they inherit their intrinsic quantum character even if they are a priori treated as classical variables. Considered from the point of view of Quantum Measurement Theory, position is reached by means of quantum fields used as probes in the measurement process [111]. Then quantum limits also emerge as a consequence of quantum field fluctuations. It is a remarkable consistency result that these two approaches agree on the ultimate limits constraining localisation in vacuum.

The quantum Brownian motion induced on position by the force fluctuations in quantum vacuum has been studied within the framework of linear response formalism [110]. For small displacements, the effects of motion on field scattering and of field radiation pressure on the scatterer's motion can be treated as reciprocal linear responses

$$
\begin{aligned}
F[\omega] & =\chi_{F F}[\omega] q[\omega]+F^{i n}[\omega] \\
q[\omega] & =\frac{1}{m\left(\omega_{0}^{2}-(\omega+i \epsilon)^{2}\right)} F[\omega]+q^{i n}[\omega]
\end{aligned}
$$

The mirror has been assumed to be anchored elastically with a proper frequency $\omega_{0}$. The solution of the linear system (24) provides the position and radiation pressure of the coupled system in terms of input fluctuations. In fact, input position fluctuations $q^{i n}$, which are localised at the proper frequencies $\pm \omega_{0}$ of the free oscillator, do not feed the final fluctuations in the coupled system, which are thus only determined by input force fluctuations $F^{i n}$. Fluctuations of positions in the coupled system are then correctly described by a quantum Langevin equation, which includes input force fluctuations $F^{i n}$ and the mirror's mechanical response function $\chi_{q q}$

$$
\chi_{q q}[\omega]=\frac{1}{m_{0}\left(\omega_{0}^{2}-\omega^{2}\right)-\chi_{F F}[\omega]}
$$

The coupled system also obeys fluctuation-dissipation relations. In particular, the noise spectrum $C_{q q}$ which characterizes position fluctuations and the susceptibility $\chi_{q q}$ which describes mechanical response of the mirror to an input force are directly connected to each other

$$
C_{q q}[\omega]=2 \hbar \theta(\omega) \xi_{q q}[\omega]=2 \hbar \theta(\omega) \operatorname{Im}\left(\chi_{q q}[\omega]\right)
$$

The noise spectrum $C_{q q}$ contains resonance peaks which correspond to the quantum fluctuations of position associated with Schrödinger's propagator for the free oscillator. In fact, these fluctuations may be seen as those fluctuations which remain in the non dissipative limit of decoupling, for a scatterer coupled to the radiation pressure fluctuations of vacuum quantum fields. Besides the resonance peaks associated with Schrödinger's propagator, the noise spectrum $C_{q q}$ includes a background which spreads over all frequencies and describes the noise added by vacuum pressure fluctuations on position fluctuations. Considering the particular case of an unbound mirror $\left(\omega_{0}=0\right)$, the background may be estimated from $(26)$ as

$$
C_{q q}[\omega] \simeq \lambda_{c}^{2} \frac{\theta(\omega)}{\omega} \quad \lambda_{C}=\frac{\hbar}{m c}
$$

where $\lambda_{C}$ is the Compton wave-length associated with mass $m$. As a consequence, an unbound mirror is shown to undergo a quantum diffusion behaviour with an order of magnitude given by the Compton wavelength [110].

The quantum nature of position can also be investigated from the point of view of Quantum Measurement Theory. Canonical commutation relations suggest that independent measurements performed at different points in space-time are constrained by a standard quantum limit 106 108. Similar constraints apply to field measurements, as early discussed by Bohr and Rosenfeld [112. The motion of the test charge used to measure the field strength results in a radiated field which perturbs a further measurement. The perturbation is given by the field propagator, that is also 
by the field commutator, between the two measurement points. Motion and field measurements are limited by the backaction of the probe field or test particle used, expressed by a response function or a commutator 111].

High sensitivity measurements of position have been reanalysed recently 113 and more emphasis has been put on the role played by the measurement strategy in fixing ultimate limitations [43, 44]. The standard quantum limit does not correspond to an optimal strategy, since it corresponds to independent successive measurements. A better exploitation of quantum correlations allows to attain lower limits [109,114 116.

This is illustrated by the example of an interferometric measurement of position, which is a prototype of a spacetime measurement of high precision [106, 117, 118]. In such a measurement, the phase $\varphi$ of a probe field is monitored. An estimation of the mirror's position $q$ is deduced ( $K_{0}$ is the mean wave-vector of the incident field)

$$
q=\frac{\varphi}{2 K_{0}}
$$

Hence, the inferred position is directly affected by phase fluctuations $\delta \varphi$ of the probe field. Simultaneously, each reflected photon of the probe field transmits twice its momentum to the mirror, so that fluctuations $\delta I$ of the photon flux $I$ in the probe field result in a fluctuating radiation pressure

$$
\delta F=2 \hbar K_{0} \delta I
$$

The mirror moves under these force fluctuations and the measured position is affected, which reveals a simple example of backaction during measurement. Finally, the measurement adds a noise $\delta q$ on the estimated position which is obtained as a linear combination of conjugate input field fluctuations (28, 29)

$$
\delta q=\frac{\delta \varphi}{2 K_{0}}+2 \hbar K_{0} \chi_{q q} \delta I \quad \chi_{q q}=\frac{1}{m\left(\omega_{0}^{2}-\omega^{2}-i \gamma \omega\right)}
$$

$\chi_{q q}$ is the mechanical susceptibility of the mirror to an applied force, assuming that it is elastically anchored at frequency $\omega_{0}$ with damping mechanisms gathered in a coefficient $\gamma$.

The phase fluctuations $\delta \varphi$ and intensity fluctuations $\delta I$ obey generalised Heisenberg inequalities at each frequency. As a result, the noise spectra associated with $\delta q$ take minimal values which fix lower bounds on the measurement sensitivity. When the probe fields have uncorrelated phase and intensity fluctuations, the lower bound is essentially determined by the modulus of the mechanical susceptibility

$$
\sigma_{\delta q \delta q}[\omega] \geq\left|\chi_{q q}[\omega]\right|
$$

This is the standard quantum limit which may be also expressed as a spectral density for the noise energy equal to Planck constant $\hbar$. A much better sensitivity is obtained [109] by using optimally squeezed fields chosen such as to minimise the fluctuations of the particular combination of the two quadrature components entering the output noise $\delta q$. Measurement sensitivity is thus limited by the dissipative part of the mechanical susceptibility

$$
\sigma_{\delta q \delta q}[\omega] \geq\left|\operatorname{Im} \chi_{q q}[\omega]\right|
$$

At the limit of a small damping $(\gamma \ll \omega)$, the dissipative part is much lower than the reactive part and the ultimate quantum limit (32) lies far beyond the standard quantum limit (31).

Fluctuations of position result from the quantum Brownian motion induced by vacuum radiation pressure fluctuations, which are also related (see (26)) to the dissipative part of the mirror's mechanical susceptibility (25). In a position measurement, the final noise will include permanent fluctuations induced by radiation pressure of vacuum fields and fluctuations added by the probe field. An optimal measurement will have its sensitivity limited by the dissipative part of the mechanical susceptibility. This dissipative part contains an irreducible component corresponding to vacuum fluctuations which sets the ultimate quantum level of sensitivity in a position measurement.

\section{GRAVITATIONAL VACUUM AND SPACE-TIME}

This ultimate noise associated with vacuum radiation pressure has an order of magnitude mainly determined by the Compton wave-length $\lambda_{C}$ associated with the mass $m$ of the scatterer (see eq.(27)). When the mass $m$ is greater than Planck mass $m_{P}$, this limit on position measurement goes beyond the Planck length $l_{P}$

$$
m_{P}=\sqrt{\frac{\hbar c}{G}} \sim 22 \mu \mathrm{g} \quad l_{P}=\sqrt{\frac{\hbar G}{c^{3}}} \sim 1.610^{-35} \mathrm{~m}
$$


where $G$ is the Newton constant. But sensitivity in length measurement is expected to be limited by Planck length in any sensible quantum theory [52,119, 120]. This implies that, at such a level of sensitivity, quantum fluctuations of gravitation must be taken into account when discussing ultimate quantum limits 121 . It is also known since Einstein [40] that metrical properties of space-time depend on the presence of a gravitational field. Localisation in space-time has thus to be affected by gravity and, particularly, by quantum fluctuations of gravity. It follows that the very notion of localisation in vacuum has to be reconsidered in view of its relation with gravitation.

Since the discovery of vacuum fluctuations, the question of their gravitational contribution has been a matter of debate. Problems with the infinite energy of vacuum fluctuations early led to the view that vacuum energy should not contribute to gravity. It was noticed that vacuum energy may be forced to vanish by definition 90,122, 123. This is done by prescribing that any expression involving quantum fields has to be normally ordered with creation and annihilation operators arranged so that the expression vanishes in vacuum. This normal ordering prescription relies on a distinction between positive and negative field frequencies which depends on the choice of coordinate map. It is therefore incompatible with the covariant description required by General Relativity. These problems are particularly acute in the context of cosmology 124 127. They cannot be solved by using a classical description of curved space as a background for quantum fields [42. Among the difficulties which arise in this domain, lies the need to regularise the infinite energy-momentum tensor [16,17]. This procedure gives rise to ambiguities and to anomalies, that is to a breakdown at the quantum level of usual symmetry properties of the energy-momentum tensor [128 131].

On the other hand, it is well known that gravitational fields can be dealt with by using standard techniques of Quantum Field Theory 48 50. However, infinities generated by gravitational radiative processes cannot be dealt with usual procedures, like renormalisation, and call for the development of a new Quantum Gravity Theory [51]. In the absence of a complete and consistent theory, discussions of the effects of quantum fluctuations of gravitational field have remained preliminary and very partial. For the same reason, different approaches rely on specific models or assumptions and they largely differ in the way to introduce gravitational quantum fluctuations.

Most approaches consider that the Einstein equations must still describe the metric structure of space-time at low energies whereas profound modifications are expected for the ultimate structure of space-time at very short distances, due to quantum fluctuations of gravitation [52]. It has been speculated that these modifications, whatever their precise form may be, should allow to remove the ultraviolet divergences of Quantum Field Theory [132, 133]. Some remarkable features, which are already present but remain exceptional in classical level, have been argued to become important at energies comparable to Planck energy. The representation of quantum evolution by means of path integrals has favored developments analysing the effects of particular configurations of classical metric, like black holes or more general topological defects, on the nature of space-time at very short distances [134]. Approximate descriptions of a fluctuating space-time have also been proposed, aiming to provide mechanisms producing corrections to standard Quantum Mechanics, localisation of classical objects or macroscopic decoherence [135 137].

Attention will be focussed in the following on a different approach which relies on a few minimal physical asumptions, deeply rooted in the conception of a space-time related to the properties of quantum vacuum. Precisely, the Einstein equation will be considered as representing the effective low energy behaviour of an underlying quantum theory. The underlying theory may have quite different forms in the limit of high frequencies where the quantum effects of gravity are expected to become predominant. These differences will not be relevant in the forthcoming discussions which depend only upon the general relations between response functions and vacuum fluctuations. Since these relations are used only at low frequencies lying much below the Planck scale, significant results can be obtained despite of the still unsolved problems of Quantum Gravity and, especially, of the problems of renormalisability [51] and of vacuum stability [138,139]. Note that attempts have been made to explain gravitation itself as the result of perturbations of vacuum energy [140,141]. In any case, quantum corrections to gravitational equations are expected to take place and they may be studied with similar assumptions [142, 143. The approach just outlined allows to derive universal noise spectra for gravitational quantum fluctuations [121]. It thus leads to a quantum structure for space-time which does not rely on any specific assumption and, in particular, does not depend on any model dependent parameter.

It has been shown from minimal physical asumptions that a quantum field theory describing gravitation by the exchange of spin 2 particles coupled to the energy-momentum tensor leads to the Einstein equation [48.49. This equation thus constitutes a firm basis for describing the propagation of gravitational fields, and hence the properties of the associated vacuum fluctuations. Because of the universal coupling of all fields to gravitation, the gravitational vacuum also affects the vacua of all other fields. The true quantum version of empty space is in fact the vacuum of quantum fields coupled to gravitational vacuum. It is therefore important that gravitational fluctuations and propagators are connected in the same manner as for any other field theory.

Proceeding in this spirit, gravitational fluctuations are described as perturbations $h_{\mu \nu}$ of Minkowski metric $\eta_{\mu \nu}$. They are given an intrinsic description, independent of a particular choice of reference system, when written in terms of space-time curvatures. The Riemann tensor $R_{\lambda \mu \rho \nu}$ is invariant under gauge transformations, i.e. changes of coordinates. It is defined in the Fourier domain at first order in the metric perturbation as 


$$
R_{\lambda \mu \rho \nu}[k]=\frac{1}{2}\left(k_{\mu} k_{\nu} h_{\rho \lambda}[k]+k_{\rho} k_{\lambda} h_{\mu \nu}[k]-k_{\mu} k_{\rho} h_{\nu \lambda}[k]-k_{\nu} k_{\lambda} h_{\mu \rho}[k]\right)
$$

The quantum fluctuations of gravitational field are then obtained from the free propagator associated with the linearised Einstein equation. When written in terms of curvatures, the corresponding noise spectrum takes a gauge independent form

$$
\begin{aligned}
C_{R_{\lambda \mu \rho \nu} R_{\lambda^{\prime} \mu^{\prime} \rho^{\prime} \nu^{\prime}}}[k] & =16 \pi^{2} l_{P}^{2} \theta\left(k_{0}\right) \delta\left(k^{2}\right) \\
& \times\left(\mathcal{R}_{\lambda \mu \lambda^{\prime} \mu^{\prime}} \mathcal{R}_{\rho \nu \rho^{\prime} \nu^{\prime}}+\mathcal{R}_{\lambda \mu \rho^{\prime} \nu^{\prime}} \mathcal{R}_{\rho \nu \lambda^{\prime} \mu^{\prime}}-\mathcal{R}_{\lambda \mu \rho \nu} \mathcal{R}_{\lambda^{\prime} \mu^{\prime} \rho^{\prime} \nu^{\prime}}\right) \\
\mathcal{R}_{\lambda \mu \rho \nu} & =\frac{1}{2}\left(k_{\lambda} k_{\rho} \eta_{\mu \nu}+k_{\mu} k_{\nu} \eta_{\lambda \rho}-k_{\mu} k_{\rho} \eta_{\lambda \nu}-k_{\lambda} k_{\nu} \eta_{\mu \rho}\right)
\end{aligned}
$$

These expressions correspond to gravitational wave zero-point fluctuations and they can be seen to be determined by Lorentz invariance of vacuum, symmetries of Riemann tensor and the vanishing of Einstein's tensor. Their status is close to that of stochastic gravitational waves which are predicted to have been generated by various cosmological or astrophysical processes [144]. As for vacuum fluctuations of the electromagnetic field, these are irreducible fluctuations, the spectral energy density of which is normalised to $\hbar \omega / 2$ per mode 145$]$.

The curvature tensor determines the gravitational effect on field propagation. The momentum of any field follows a law of geodesic deviation, and thus integrates curvature perturbations encountered along propagation. This geodesic deviation takes the same expression as the frequency shift induced by a classical background gravitational wave 146 148. When integrated along the propagation of the field, curvature fluctuations induce fluctuations of propagation distances. These geodesic fluctuations affect space-time localisation, which uses probe fields, as well as the definition of vacuum of quantum fields. For space-time localisation, the fluctuations induced on the probe field result in a noise spectrum for measured lengths or positions [121]. For frequencies higher than the inverse of the propagation time, the noise spectrum is shown to take a universal form

$$
C_{q q}[\omega] \simeq l_{P}^{2} \frac{\theta(\omega)}{\omega}
$$

with a further numerical factor depending on the particular measurement technique used, for instance one-way or round trip probing. Curvature fluctuations then impose a limit on space-time probing of the order of Planck length. The spectrum of fluctuations in vacuum (36), which holds for frequencies smaller than Planck frequency $\frac{c}{l_{P}}$ has been deduced from the minimal assumptions discussed previously, namely an effective behavior of gravitation at low frequencies given by Einstein equation, and a conformity of vacuum fluctuations with fluctuation-dissipation relations. This spectrum exhibits the characteristic properties of vacuum correlations, with fluctuations (36) restricted to positive frequencies. Hence, a commutator spectrum is obtained from (36), which implies that geodesic distances are noncommutative and behave as quantum variables. Thus, the minimal assumptions just mentioned lead to behaviours characterising an underlying non-commutative geometry. This pleads for a drastic renouncement to the classical nature of space-time and, accordingly, to the associated classical geometry. Note that quantum generalisations of geometry have already been proposed where the symmetry groups associated with classical geometry are replaced by quantum groups [149 153]. It has to be emphasized that the predictions of the approach sketched here does not depend on any free parameter. They may hence be used to select among the many candidates those generalisations of classical geometry which fit the Einstein equation while making gravitational vacuum fluctuations conform to fluctuation-dissipation relations.

Fluctuations of geodesic distance induced by vacuum gravitational waves take the same form as fluctuations of position (27) induced by radiation pressure of electromagnetic vacuum. The order of magnitude is however different since it is here determined by the Planck length and not by the Compton wave-length. In particular, fluctuations of geodesic distances do not depend on the mass of the mirror. In a position measurement performed with a probe field, two different regimes of ultimate quantum noise are found, depending on the endpoint mass used. For a "microscopic" mass, smaller than the Planck mass, radiation pressure fluctuations dominate. But for a "macroscopic" mass, greater than the Planck mass, the ultimate quantum noise no longer depends on the test mass used and must be attributed to fluctuations of space-time itself. Geometric fluctuations of quantum space-time are probed only when macroscopic endpoint reflectors are used. These results suggest that a natural borderline might be delineated between the macroscopic and microscopic world provided that quantum fluctuations of gravity are accounted for.

We have discussed in the present section how massless fields allow to probe light-like space-time intervals. Massive probe fields can also be used, as is the case for instance in atomic interferometers [154]. They would allow to test timelike intervals [81]. We may also note that effects of gravitation vacuum fluctuations subsist for quantum fields which are themselves in vacuum. Indeed, vacuum field correlations depend on the geodesic distance which characterize the relative positions of two points in space-time. On the other hand, vacuum field fluctuations carry energy-momentum fluctuations which are themselves a cause for metric fluctuations. As a result, the gravitational and non-gravitational vacua must be treated as coupled systems [81]. 


\section{CONCLUSION AND PERSPECTIVES}

We have seen that vacuum fluctuations have a profound impact on fundamental concepts of mechanics and of relativity. In particular, the principle of relativity of motion is directly connected to the symmetries of vacuum. This connection was well-known for the cases of uniform velocity and Lorentz invariance and it has to be extended to uniform acceleration and conformal symmetry. As soon as non uniform acceleration is involved, motion in vacuum gives rise to radiation reaction forces opposed to the motion.

In order to appreciate the significance of these results, it is worth recalling the difference between the covariance properties which constitute the basement of General Relativity and the invariance properties which are at the heart of the Special Theory of Relativity [155]. Clearly, the connection between vacuum symmetries and relativity of motion lies on the side of invariance properties. To go further in the same direction, it may be considered that this connection challenges the general principle of relativity, that is the principle of relativity of arbitrary motion. At this point, two different opinions may be upheld. On one hand, it may be hoped that a future theory of Quantum Gravity will cure this difficulty and revive the general principle of relativity at the quantum level. On the other hand, it may be considered that uniform velocity and uniform acceleration effectively play a privileged role since they represent the only frictionless motions in vacuum. In any case, the concept of relativity of motion should play a key role in the development of theoretical frameworks consistently dealing with quantum fluctuations and gravitational phenomena.

The same question also plays a central role in the construction of a concept of space-time accounting for quantum fluctuations as well as for relativistic symmetries. As well-known since the advent of Relativity Theory, time and space can no longer be considered as absolute notions as it was the case in the Newtonian framework. The basic element of a relativistic conception of space-time is that of events occuring at some position in space and time [3]. Comparison of events occuring at different locations is performed through clock synchronisation procedures built on the transfer of electromagnetic signals. These ideas have now been included in metrological definitions of time and space [156]. At some high level of precision, synchronisation procedures have to reach a limit associated with the quantum nature of the signals used in the transfer 157,158]. More profoundly, space-time observables associated with a given event certainly belong to the quantum domain, like atomic clocks used for time definition and electromagnetic signals used for synchronisation. As a consequence, the shifts of space-time observables under transformations to accelerated frames cannot be deduced from covariance properties associated with the corresponding map transformations. It has recently been shown that these questions may be given satisfactory answers by using the same symmetries which have been used here to represent the properties of motion. The conformal algebra indeed determines the conserved quantities. For field states with a non vanishing mass, such as those used to perform the Einstein localisation of an event in space-time, these quantities may be used to define quantum observables associated with positions of an event in space-time [159]. The conformal algebra also fixes the shift of these observables under changes of reference frames and thus describes within a quantum framework the relativistic effects associated with uniform and accelerated motions. The shift of mass under transformations to accelerated frames may be written in terms of position observables, in consistency with Einstein principle of equivalence [160].

Space-time observables conceptually differ from coordinate parameters, as emphasized by their relativistic and quantum properties. In particular, a change of reference frame has to be distinguished from a change of coordinate map, since the former is directly related to relativistic symmetries whereas the second is a mere matter of convention. The problem is particularly acute for the definition of time which has been thoroughly discussed in connection with fundamental questions of Quantum Measurement Theory [161] and Quantum Gravity [162]. A time observable associated with the position of an event is defined, besides space observables, by the procedure just described. It is however a localisation observable which differs from any kind of evolution parameter. A lasting challenge is hence to represent movement in a quantum framework where the prime roles are played by conserved quantities [163 166 . It is amazing that this paradox of modern physics lies in the continuity of the logical debates of the ancient philosophers. It might be that its solution requires a new definition of movement which should be conceived, in the spirit of the atomistic line of thought, as a sequence of events [167].

Acknowledgements Thanks are due to Vladimir Braginsky, Jean-Michel Courty, Leonid Grishchuk, Astrid Lambrecht, Paolo Americo Maïa Neto, Roberto Onofrio, Carlo Rizzo and Lorenza Viola for stimulating discussions.

[1] Russell B. 1961 History of Western Philosophy (London: George Allen \& Unwin; reprinted by Routledge 1991) 
[2] Koyré A. 1957 From the Closed World to the Open Universe (Baltimore: JohnHopkins Press)

[3] Einstein A. 1946 The Meaning of Relativity (Princeton: Princeton University Press)

[4] Einstein A. 1905 Ann. Physik 18 639; 1906 Ann. Physik 20627

[5] Einstein A. 1905 Ann. Physik 17 549; 1909 Phys. Z. 10 185; 1917 Phys. Z. 18121

[6] Planck M. 1900 Verh. Deutsch. Phys. Ges. 2237

[7] Planck M. 1911 Verh. Deutsch. Phys. Ges. 13138

[8] Einstein A. and Stern O. 1913 Ann. Physik 40551

[9] Nernst W. 1916 Verh. Deutsch. Phys. Ges. 1883

[10] Dirac P. A. M. 1958 The Principles of Quantum Mechanics (Oxford: Oxford University Press)

[11] Sciama D. W. 1991 in The Philosophy of Vacuum eds S. Saunders and H.R. Brown (Oxford: Clarendon)

[12] Boyer T. 1973 Phys. Rev. D 11790

[13] Rindler W. 1966 American Journal of Physics 34 1174; 1969 Essential Relativity (New York: Van Nostrand Reinhold)

[14] Davies P. C. W. 1975 Journal of Physics A 8609

[15] Unruh W. G. 1976 Phys. Rev. D 14870

[16] Birrell N. D. and Davies P. C. W. 1982 Quantum Fields in Curved Space (Cambridge: Cambridge University Press)

[17] Fulling S. A. 1989 Aspects of Quantum Field Theory in Curved Spacetime (Cambridge: Cambridge University Press)

[18] Zel'dovich Ya. B. and Starobinsky A. A. 1971 Zh. Eksp. Teor. Fiz. 61216 [Sov. Phys. JETP 34 1159]; 1977 Pisma Zh. Eksp. Teor. Fiz. 26373 [Sov. Phys. JETP Lett. 26 252]

[19] Hawking S. W. 1975 Comm. Math. Phys. 43199

[20] Moore G. T. 1970 J. Math. Phys. 112679

[21] de Witt B. S. 1975 Phys. Rep. 19295

[22] Fulling S. A. and Davies P. C. W. 1976 Proc. R. Soc. A 348393

[23] Einstein A. 1907 Jahrb. Radioakt. Elektron. 4 411; 1908 Jahrb. Radioakt. Elektron. $598 ; 1911$ Ann. Physik 35898

[24] Bateman H. 1909 Proc. London Math. Soc. 7 70; ibid. 8223

[25] Cunningham E. 1909 Proc. London Math. Soc. 877

[26] Itzykson C. and Zuber J. B. 1985 Quantum Field Theory (New York: McGraw Hill)

[27] Special Issue on "Squeezed Light" eds R. Loudon and P. Knight 1987 Journal of Modern Optics 34 709-1020

[28] Special Issue on "Squeezed States of the Electromagnetic Field" eds J. Kimble and D. Walls 1987 Journal of the Optical Society of America B 4 1449-1741

[29] Special Issue on "Quantum Noise Reduction in Optical Systems" eds E. Giacobino and C. Fabre 1992 Applied Physics B 55 189-303

[30] Reynaud S., Heidmann A., Giacobino E. and Fabre C. 1992 in Progress in Optics XXX ed. E. Wolf (Amsterdam: North Holland) 1

[31] Casimir H. B. G. 1948 Proc. K. Ned. Akad. Wet. 51793

[32] Plunien G., Müller B. and Greiner W. 1986 Physics Reports 13487

[33] Mostepanenko V. M. and Trunov N. N. 1988 Uspekhi Fisica Nauk 156385 [Sov. Phys. Uspekhi 31 965]

[34] Deriagin B. V. and Abrikosova I. I. 1956 Jh. Eksp. Teor. Fis. 30993 [Soviet Physics JETP 3 819]

[35] Sparnaay M. J. 1958 Physica XXIV 751; Black W., De Jongh J. G. V., Overbeek J. T. G. and Sparnaay M. J. 1960 Transactions of the Faraday Society $\mathbf{5 6} 1597$

[36] Tabor D. and Winterton R. H. S. 1968 Nature 2191120

[37] Sabisky E. S. and Anderson C. H. 1973 Physical Review A 7790

[38] Lamoreaux S. K. 1997 Phys. Rev. Lett. 785

[39] Sukenik C. I., Boshier M. G., Cho D., Sangdohar V. and Hinds E. A. 1993 Phys. Rev. Lett. 70560

[40] Einstein A. 1916 Ann. Physik

[41] Rosenfeld L. 1963 Nuclear Physics 40353

[42] de Witt B. S. 1962 J. Math. Phys. 3619

[43] Braginsky V. B. and Khalili F. Ya. 1992 Quantum Measurement (Cambridge: Cambridge University Press)

[44] Bocko M. F. and Onofrio R. 1996 Reviews of Modern Physics 68755

[45] Blohincev D. I. 1960 Nuovo Cimento 61382

[46] Gupta S. N. 1952 Proc. Phys. Soc. 65 161, 608

[47] Utiyama R. and de Witt B. S. 1962 J. Math. Phys. 3608

[48] Feynman R. P. 1963 Acta Physica Polonica 247118

[49] Weinberg S. 1965 Phys. Rev. 138 B988

[50] Zel'dovich Ya. B. and Grishchuk L. P. 1986 Uspekhi Fisica Nauk 149695 [Sov. Phys. Uspekhi 29 780]

[51] t'Hooft G. and Veltman M. 1974 Ann. Inst. Henri Poincaré 2069

[52] Wheeler J. A. 1957 Ann. Phys. 2604

[53] Rovelli C. 1991 Class. Quantum Grav. 8 297, 317

[54] Isham C. J. 1995 "Structural Issues in Quantum Gravity" to appear in the Proceedings of the GR14 conference grqc/9510063]

[55] Jaekel M. T. and Reynaud S. 1995 Quantum Semiclass. Opt. 7499 
[56] Jaekel M. T. and Reynaud S. 1995 Brazilian J. Phys. 25325

[57] Callen H. B. and Welton T. A. 1951 Phys. Rev. 8334

[58] Kubo R. 1966 Rep. Prog. Phys. 29255

[59] Born M. 1909 Ann. Physik 301

[60] Unruh W. G. and Wald R. M. 1984 Phys. Rev. D 291047

[61] Unruh W. G. 1992 Phys. Rev. D 463271

[62] Bell J. S. and Leinaas J. M. 1983 Nucl. Phys. B 212131

[63] Rogers J. 1988 Phys. Rev. Lett. 612113

[64] Rosu H. C. 1994 Int. J. of Modern Phys. D 3545

[65] Grove P. G. 1986 Class. Quantum Grav. 3 793; 1988 Class. Quantum Grav. 51381

[66] Raine D. J., Sciama D. W. and Grove P. G. 1991 Proc. R. Soc. London A 435205

[67] Audretsch J. and Müller R. 1994 Phys. Rev. D 496566

[68] Ginzburg V. L. and Frolov V. P. 1987 Usp. Fiz. Nauk 153633 [Sov. Phys. Usp. 30 1073]

[69] Hill W. L. 1945 Phys. Rev. 67 358; 1947 Phys. Rev. 72143

[70] Page L. 1936 Phys. Rev. 49 254; Page L. and Adams N. L. 1936 Phys. Rev. 49466

[71] Gupta S. N. 1961 Science 1341360

[72] Fulton T., Rohrlich F. and Witten L. 1962 Rev. Mod. Phys. 34 442; 1962 Nuovo Cimento 26653

[73] Dirac P. A. M. 1936 Annals of Math. 37429

[74] Bhabha H. J. 1936 Proceedings of the Cambridge Philosophical Society 32622

[75] Itzykson C. and Drouffe J. M. 1989 Statistical Field Theory (Cambridge: Cambridge University Press)

[76] Ford L. H. and Vilenkin A. 1982 Phys. Rev. D 252569

[77] Maia Neto P. A. 1994 J. Phys. A Math. Gen. 272167

[78] Jaekel M. T. and Reynaud S. 1992 Quantum Opt. 439

[79] Jaekel M. T. and Reynaud S. 1993 J. Phys. France I 31093

[80] Barton G. 1991 J. Physics A 24 991, 5533; 1994 Cavity Quantum Electrodynamics (Supplement: Advances in Atomic, Molecular and Optical Physics) ed. P. Berman (New York: Academic Press)

[81] Jaekel M. T. and Reynaud S. 1995 Ann. Physik 468

[82] Brown L. S. and Maclay G. J. 1969 Phys. Rev. 1841272

[83] Jaekel M. T. and Reynaud S. 1991 J. Phys. France I 11395

[84] Iacopini E. 1993 Phys. Rev. A 48129

[85] Lambrecht A., Jaekel M. T. and Reynaud S. 1997 Phys. Lett. A 225188

[86] Barton G. 1963 Introduction to Advanced Field Theory (New York: Interscience Publishers)

[87] Barton G. and Eberlein C. 1993 Ann. Phys. 227222

[88] Eberlein C. 1992 J. Phys. A 25 3015, 3039

[89] Maia Neto P. A. and Reynaud S. 1993 Phys. Rev. A 471639

[90] Pauli W. 1921 Relativitätstheorie: Encyklopädie des Wissenschaften V 19 (Leipzig: B. G. Teubner) [1958 Theory of Relativity (New York: Pergamon Press)]

[91] Dekker H. 1985 Phys. Lett. A 107 255; 1985 Physica 133 A 1

[92] Ford G. W., Lewis J. T. and O'Connell R. F. 1985 Phys. Rev. Lett. 552273

[93] Cohen-Tannoudji C., Dupont-Roc J. and Grynberg G. 1988 Processus d'interaction entre photons et atomes (Paris: InterEditions)

[94] Milonni P. W. 1994 The Quantum Vacuum (San Diego: Academic Press)

[95] Rohrlich F. 1965 Classical Charged Particles (Reading: Addison-Wesley)

[96] Jaekel M. T. and Reynaud S. 1992 Phys. Lett. A 167227

[97] Dodonov V. V., Klimov A. B. and Man'Ko V. I. 1989 Phys. Lett. A 142 511; 1990 Phys. Lett. A 149 225; Dodonov V. V. 1995 Phys. Lett. A 207126

[98] Sassaroli E., Srivastava Y. N. and Widom A. 1994 Phys. Rev. A 501027

[99] Braginsky V. B. and Khalili F. Ya. 1991 Phys. Lett. A 161197

[100] Lambrecht A., Jaekel M. T. and Reynaud S. 1996 Phys. Rev. Lett. 77615

[101] Jaekel M. T. and Reynaud S. 1993 J. Phys. France I 3339

[102] Jaekel M. T. and Reynaud S. 1993 Phys. Lett. A 1809

[103] Haroche S. 1984 in New Trends in Atomic Physics, eds G. Grynberg and R. Stora (Amsterdam North Holland) 193

[104] Law C. K. 1994 Phys. Rev. Lett. 731931

[105] Cole and Schieve 1995 Phys. Rev. A 524405

[106] Caves C. M. 1980 Phys. Rev. Lett. 45 75; 1981 Phys. Rev. D 23 1693; 1985 Phys. Rev. Lett. 542465

[107] Yuen H. P. 1983 Phys. Rev. Lett. 51719

[108] Ozawa M. 1988 Phys. Rev. Lett. 60 385; 1990 Phys. Rev. A 411735

[109] Jaekel M. T. and Reynaud S. 1990 Europhys. Lett. 13301

[110] Jaekel M. T. and Reynaud S. 1993 J. Phys. France I 31

[111] de Witt B. S. 1963 in Relativity, Groups and Topology eds C. de Witt and B. de Witt (New York: Gordon Breach) 
[112] Bohr N. and Rosenfeld L. 1950 Phys. Rev. 78794

[113] Braginsky V. B. 1988 Usp. Fiz. Nauk 15693 [Sov. Phys. Usp. 31 836]

[114] Unruh W. G. 1983 Quantum optics, experimental gravitation and measurement theory eds. P. Meystre and M. O. Scully (New York: Plenum) 647

[115] Luis A. and Sánchez-Soto L. L. 1992 Phys. Rev. A 458228

[116] Pace A. F., Collett M. J. and Walls D. F. 1993 Phys. Rev. A 473173

[117] Grangier P., Slusher R. E., Yurke B. and LaPorta A. 1987 Phys. Rev. Lett. 592566

[118] Min Xiao, Wu L. A. and Kimble H. J. 1987 Phys. Rev. Lett. 592781

[119] de Witt B. S. 1962 in Gravitation, an introduction to current research ed. L. Witten (New York: Wiley) 266

[120] Padmanabhan T. 1987 Class. Quant. Gravity 4 L107

[121] Jaekel M. T. and Reynaud S. 1994 Phys. Lett. A 185143

[122] Feynman R. P. and Hibbs A. R. 1965 Quantum Mechanics and Path Integrals (New York: McGraw Hill) p. 244

[123] Enz C. P. 1974 in Physical Reality and Mathematical Description eds C. P. Enz and J. Mehra (Dordrecht: Reidel)

[124] Zel'dovich Ya. B. 1981 Usp. Fiz. Nauk 133479 [Sov. Phys. Usp. 24 216]

[125] Weinberg S. 1989 Rev. of Modern Phys. 611

[126] Wesson P. S. 1991 Astrophys. J. 378466

[127] Adler R. J., Casey B. and Jacob O. C. 1995 American J. of Phys. 63620

[128] Christensen S. M. 1976 Phys. Rev. D 142490

[129] Dowker J. S. and Critchley R. 1977 Phys. Rev. D 163390

[130] Wald R. M. 1978 Annals of Physics 110 472; 1978 Phys. Rev. D 171477

[131] Horowitz G. 1980 Phys. Rev. D 211445

[132] de Witt B. S. 1964 Phys. Rev. Lett. 13114

[133] Isham C. J., Abdus Salam and Strathdee J. 1971 Phys. Rev. D 31805

[134] Hawking S. W. 1987 Phys. Lett. 195337

[135] Karolyhazy F. 1966 Nuovo Cimento A 421506

[136] Ellis J., Mohanty S. and Nanopoulos D. V. 1989 Phys. Lett. B 221113

[137] Percival I. C. 1995 Proc. R. Soc. London A 451503

[138] Horowitz G. T. and Wald R. M. 1978 Phys. Rev. D 17414

[139] Hartle J. B. and Horowitz G. T. 1981 Phys. Rev. D 24257

[140] Sakharov A. D. 1967 Doklady Akad. Nauk SSSR 17770 [Sov. Phys. Doklady 12 1040]

[141] Adler R.J. 1982 Rev. Modern Phys. 54729

[142] Donoghue J. F. 1994 Phys. Rev. Lett. 722996

[143] Ford L. H. 1995 Phys. Rev. D 511692

[144] Grishchuk L. P. 1977 Uspekhi Fisica Nauk 121629 [Sov. Phys. Uspekhi 20 319]; 1988 Uspekhi Fisica Nauk 156297 [Sov. Phys. Uspekhi 31 940]

[145] Grishchuk L. P. and Sidorov Y. V. 1990 Phys. Rev. D 423413

[146] Sachs R. K. and Wolfe A. M. 1967 Astrophys. J. 14773

[147] Braginsky V. B. and Mensky M. B. 1971 Pisma Zh. Eksp. Teor. Fiz. 13585 [Sov. Phys. JETP Lett. 13 417]

[148] Mashhoon B. and Grishchuk L. P. 1980 Astrophys. J. 236990

[149] Woronowicz L. 1987 Commun. Math. Phys. 111 613; 1989 Commun. Math. Phys. 122125

[150] Majid S 1988 Class. Quantum Grav. 51587

[151] Lukierski J. and Ruegg H. 1991 Phys. Lett. B 264331

[152] Connes A. 1995 in Gravitation and Quantizations eds B. Julia and J. Zinn-Justin (Amsterdam: Elsevier) 805

[153] Kempf A. 1997 J. of Math. Phys. to appear hep-th/9602085

[154] Bordé C. 1997 in Atomic Interferometry ed. P. Berman (Academic Press) to appear

[155] Norton J. D. 1993 Rep. Progr. Phys. 56791

[156] Special Issue on "Time and Frequency" 1991 eds J. Jespersen and D. W. Hanson Proceedings of IEEE 79 891-1079

[157] Newton T. D. and Wigner E. P. 1949 Review of Modern Physics 21400

[158] Salecker H. and Wigner E. P. 1958 Phys. Rev. 109571

[159] Jaekel M. T. and Reynaud S. 1996 Phys. Rev. Lett. 76 2407; Phys. Lett. A220 10

[160] Jaekel M. T. and Reynaud S. 1997 EuroPhys. Lett. 381

[161] Jammer M. 1974 The Philosophy of Quantum Mechanics (Wiley) ch.5

[162] Isham C.J. 1993 in Integrable Systems, Quantum Groups and Quantum Field Theories (Kluwer) 157

[163] Page D. N. and Wootters W. K. 1983 Phys. Rev. D 272885

[164] Unruh W. G. 1989 Int. J. of Modern Phys. 281181

[165] Rovelli C. 1991 Phys. Rev. D 43 442; 1995 Nuovo Cimento 110 B 81

[166] Jaekel M. T. and Reynaud S. 1997 "Conformal symmetry and quantum relativity" preprint LPTENS 97/23

[167] Russell B. 1925 ABC of Relativity (London: George Allen \& Unwin; reprinted by Routledge 1993) 